\title{
New insight on the cycloaddition of aryl and heteroaryl azides with (trimethylsilyl)acetylene. Spectroscopic and $x$-ray crystallographic data of silylated 1,2,3-triazoles
}

\author{
Silvia Businelli ${ }^{a}$, Emanuela Di Martino ${ }^{b}$, and Paolo Zanirato ${ }^{a}$ \\ ${ }^{a}$ Dipartimento di Chimica Organica 'A. Mangini', Università degli Studi di Bologna \\ ${ }^{\boldsymbol{b}}$ Dipartimento di Chimica Fisica ed Inorganica, Università degli Studi di Bologna, Viale \\ Risorgimento 4, 40136 Bologna (Italy) \\ E-mail: zanirato@ms.fci.unibo.it
}

(received 13 Jan 01; accepted 08 Nov 01; published on the web 16 Nov 01)

\begin{abstract}
Reactions of the dipolarophile (trimethylsilyl)acetylene with a number of aryl 1a - f and heteroaryl azides $\mathbf{1 g}$ - i have been examined as routes to the 1-aryl (or 1-heteroaryl) trimethylsilyl-1,2,3-triazoles 2a - i. Regioselectivity that privileges the formation of the corresponding C-4 silylated triazoles, over the C-5 ones was observed in all instances. The regiochemistry of the $\mathrm{C}-4$ adducts was established by ${ }^{1} \mathrm{H}-,{ }^{13} \mathrm{C}-\mathrm{NMR}$ spectroscopy and confirmed by X-ray crystallography of the 2,2,2-trifluoro-1-\{5-[4-(trimethylsilyl)-1H-1,2,3-triazol-1-yl]-2thienyl $\}$-1-ethanone $2 \mathbf{i}$ and 1-phenyl-4-(trimethylsilyl)-1H-triazole $2 \mathbf{l}$.
\end{abstract}

Keywords: Organic azides, silylated 1,2,3-triazoles, 1,3-dipolar cycloaddition, regiochemistry, X-ray crystallography

\section{Introduction}

In a previous paper we described the reaction of some aryl azides and the 2- and 3azidobenzo[b]thiophenes with (trimethylsilyl)acetylene to give 1-aryl (or 1-heteroaryl)-4trimethylsilyl-1,2,3-triazoles via 1,3-dipolar cycloaddition (1,3-DC). ${ }^{1}$ High yields of C-4 silylated triazoles as the unique isomer were obtained in all instances. Owing the practical applications of triazoles, for examples in agriculture and medicine ${ }^{2}$, the 1,3 -DC provides the most useful route to triazole systems containing a large number of different functionalities. ${ }^{3}$

In the cases of terminal (or asymmetrically substituted) dipolarophiles, regioselectivity is an 
essential feature of the reaction mechanism that has long been the core topic for theoretical chemists. ${ }^{4}$ Normally, when steric effects are irrelevant the organic azide adds concertedly to the dipolarophile under the control of the frontier molecular orbital (FMO) with the possible formation of two isomers. Conversely, in the presence of severe steric hindrance at one of the end of the dipole (and/or the dipolarophile) stepwise cycloadditions can compete with the concerted process. Theoretical groundwork based on FMO theory ${ }^{5}$ and, more recently, on electronic structure calculations ${ }^{6}$ has been applied to explain the regiochemistry in 1,3-DC.

Further preliminary studies uncovered that some five-membered heteroaryl azides ${ }^{7}$ react smoothly at room temperature with (trimethylsilyl)acetylene preferentially leading to the formation of C-4 silylated 1,2,3-triazoles. Electronic and steric factors operating in the same direction might be responsible for the high regioselectivity observed in the cases studied. ${ }^{1}$ Our interest in this topic is focussed on the orientation of the cycloaddition of different azides fixing as a probe the dipolarophile (trimethylsilyl)acetylene. At the same time, we are interested in the possible activity against Mycobacterium avium and tuberculosis of the resulting silylated 1,2,3triazoles. ${ }^{8}$ These activity properties can be ascertained by using the large number of cycloaddition reactions available.

In the present work we report experimental evidences on the regiochemistry of 1,2,3-triazoles arising from 1,3-DC of a number of fluorinated (fluoro and trifluoromethyl) aryl azides 1a - f and para-like substituted heteroaryl azides $\mathbf{1} \mathbf{g}$ - $\mathbf{i}$ in neat (trimethylsilyl)acetylene.

The orientation of triazoles $\mathbf{2 a}-\mathbf{i}$ is confirmed by ${ }^{1} \mathrm{H}$ - and ${ }^{13} \mathrm{C}-\mathrm{NMR}$ spectroscopic data and by comparison with the structures of 1-phenyl-4-(trimethylsilyl)-1H-1,2,3-triazole $2 \mathbf{l}$ and 2,2,2trifluoro-1-\{5-[4-(trimethylsilyl)-1H-1,2,3-triazol-1-yl]-2-thienyl\}-1-ethanone $2 \mathbf{i}$ which have been definitely established by X-ray crystallography.

\section{Results and Discussion}

The transformation of aryl and heteroaryl azides $1 \mathbf{a}-\mathbf{i}$ to 1,2,3-triazoles $\mathbf{2 a}-\mathbf{i}$ by 1,3-DC occurs according to Scheme 1. In particular, the reactions of aryl azides $\mathbf{1 a}-\mathbf{f}$ in the presence of neat (trimethylsilyl)acetylene proceed smoothly at room temperature to afford the corresponding silylated triazoles 2a - f in high yields. (Scheme 1 and Table 1, entries 1 - 6) Reactions were carried out in the dark and were completed within 20-40 days (until TLC showed disappearance of the starting azide). The excess solvent-reagent was eliminated under vacuum and the residue analysed by NMR. Conversion of all these azides into C-4 silylated triazoles was found by NMR spectroscopy to be regiospecific, with the resulting spectral data fully consistent with the proposed structures (Tables 1 and 2). The H-5' chemical shifts of the triazole ring range at $7.05-$ 
$8.05 \mathrm{ppm}$ and the 13C NMR spectra exhibit characteristic signals for C-4 and C-5 of the triazole ring at 146-150 ppm and 125-132 ppm (JC-H 192-194 Hz), respectively $2 a$.

A number of studies have been devoted to the correlation between the chemical shifts and coupling constants of protons, carbon, and fluorine atoms in substituted aryl and heteroaryl derivatives, because of the well defined electrical effects of the substituent in such systems. ${ }^{9}$ The 1-phenyl-4-(trimethylsilyl)-1H-triazole 2l was utilized to determine the substituent chemical shifts (SCS) produced by the triazole ring on the aromatic carbon, while the effects of fluorine atom and trifluoromethyl group were from the literature. ${ }^{9 \mathrm{a}, \mathrm{c}, \mathrm{d}}$ The $\mathrm{C}-1$ signals of the phenyl ring range at 135-140 ppm except for the ortho-fluoro derivative 2d (125.5 ppm; Table 2). Comparatively larger deviations between the measured and the calculated 13C NMR shifts were observed for ortho-substituted derivatives, particularly for the fluorine substituent. Nevertheless the average error of the global SCS prediction was $<1$ ppm (n $32 r$ 0.998).9a The structure of $2 \mathbf{d}$ would suffer from restricted rotation about the $\mathrm{C} 1-\mathrm{N} 1$ bond. That is, the molecule has its lowest energy in a planar conformation in which the orbitals of the fluorine atom have the greatest overlap with the adjacent $\mathrm{H}-5^{\prime}$ orbital. It is of interest to note the high conjugation of the molecule 2d evidence also by the heteronuclear coupling (JH-F $2.9 \mathrm{~Hz})$ between the fluorine atom and the $\mathrm{H}-5^{\prime}$. 


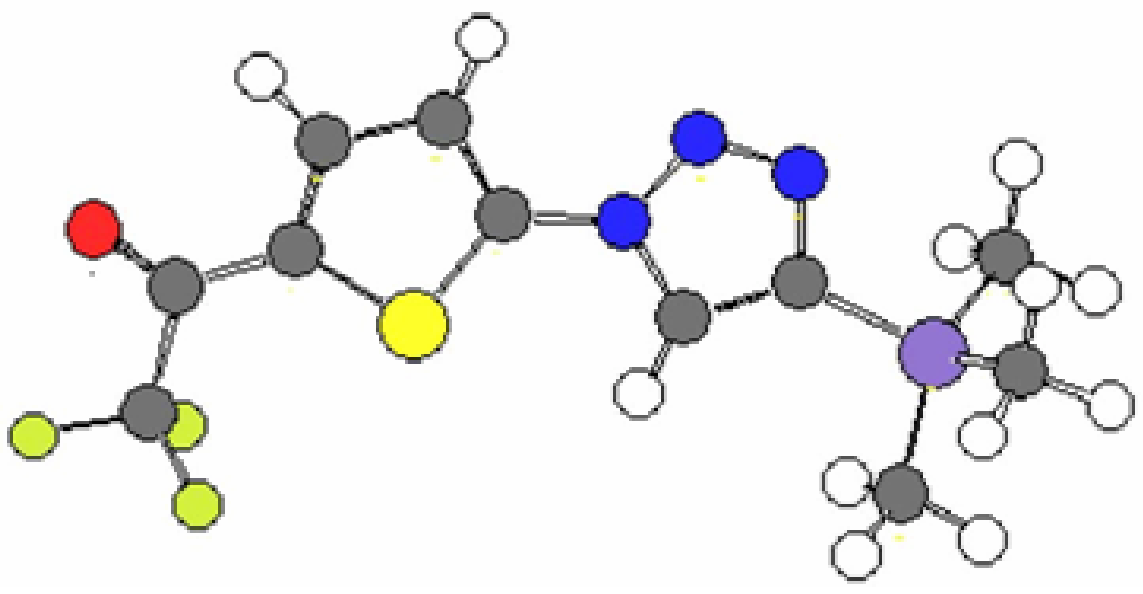

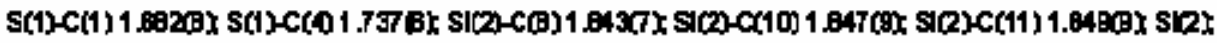

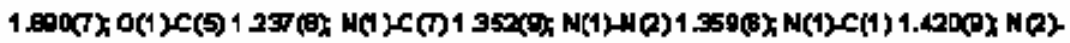
$N(3) C(\theta) 1.374(\theta) F(1) C(\theta) 1.335(1) \times F(2) C(8) 1.321$ (11) $F(3)-C(8) 1.305(10) C(1)-C(2) 1.373(\theta) C(2)$

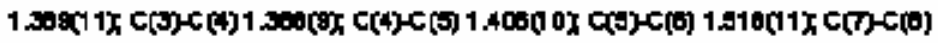

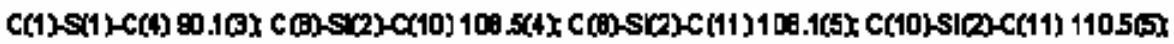

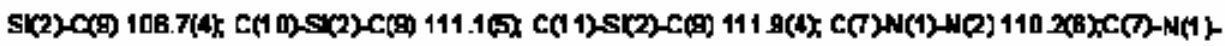

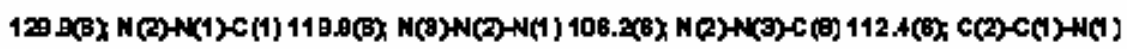

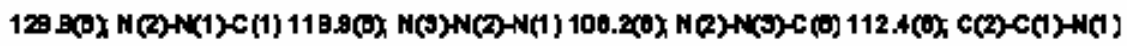

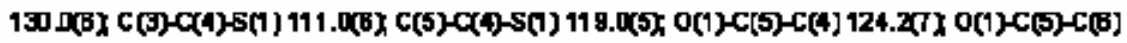

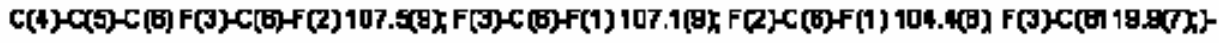

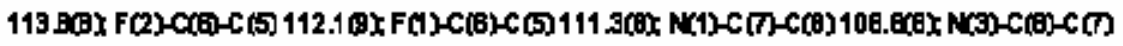
M(9) C(B) $5(2) 1253(5), C(7) C(\theta) S(2)$

Scheme 1. Bond lengths $(\AA)$ and angles $\left(^{\circ}\right)$ for 2,2,2-trifluoro-1-\{5-[4-( H1,2,3-triazol-1-yl]- 2 thienyl $\}$-1-ethanone.

Analogous 1,3-DC with heteroaryl azide para-like substituted such as 5-(trimethylsilyl)-2selenophenyl azide 1g, 1-(5-azido-2-thienyl)-1-ethanone $\mathbf{1 h}$ and 2,2,2-trifluoro-(5-azido-2thienyl)-1-ethanone $1 \mathbf{i}$ proceeded faster at room temperature. The reactions afford in 10, 6 and 5 days (tlc), respectively, precipitates of the corresponding solid cycloadducts; such as 4(trimethylsilyl)-1-[5-(trimethylsilyl)-2-selenophenyl]-1H-1,2,3-triazole $\quad 2 g, \quad$ 1-\{5-[4(trimethylsilyl)-1H-1,2,3-triazol-1-yl]-2-thienyl $\}-1$-ethanone (97\%) 2h and 2,2,2-trifluoro-1-\{5[4-(trimethylsilyl)-1H-1,2,3-triazol-1-yl]-2-thienyl\}-1-ethanone 2i (Table 1, entries 7 - 9). In this latter case the major isomer $2 \mathbf{i}(97 \%)$ was obtained together with small amounts of the C-5 silylated 2,2,2-trifluoro-1-\{5-[5-(trimethylsilyl)-1H-1,2,3-triazol-1-yl]-2-thienyl $\}$-1-ethanone 2i' (Table 1, entry 9). IR, ${ }^{1} \mathrm{H}-$, and ${ }^{13} \mathrm{C}-\mathrm{NMR}$ (Table 2) spectroscopy confirmed structural assignments for all new isolated triazoles $2 \mathbf{a}-\mathbf{i}$. 
To confirm the regiochemistry of C- 4 silylated derivatives $\mathbf{2 i}$ and 2 l, previously prepared from phenyl azide 11, ${ }^{1}$ we made an effort to obtain single crystals suitable for X-ray crystallography. The crystal structure definitely confirms the regiochemistry of $\mathbf{2 i}$ and $\mathbf{2 l}$, whose significant bond length $(\AA)$ and angle $\left(^{\circ}\right)$ are reported in Figure 1 and 2.

In a preliminary communication ${ }^{7 a}$ we reported that a similar reaction, entailing 5(trimethylsilyl)-2-thienyl azide 1m was completed within 7 days leading to the exclusive formation of the C-4 silylated triazole, i.e. 4-(trimethylsilyl)-1-[5-(trimethylsilyl)-2-thienyl]-1H1,2,3-triazole $\mathbf{2} \mathbf{m}$. Conversion of this azide was found by NMR spectroscopic analysis to be completely regiospecific. On the other hand, the reaction of 2-azido-5-methylthiophene $1 \mathrm{n}$ was virtually completed after 7 days, but at $+5{ }^{\circ} \mathrm{C}$. Mixture of the C-4 and C-5 isomers (2n : 2n'; 1 : 0.05 ) were obtained accompanied by small amounts of ring opening product, due to the low stability of the starting azide 1n making it unable to survive longer under the reaction conditions. ${ }^{10}$ In summary, we found general evidence that the 1,3-DC of aryl and heteroaryl azides to (trimethylsilyl) acetylene is a FMO and steric controlled process, resulting in the preferential formation of C-4 silylated triazoles. From our findings we infer that steric hindrance plays an important role in increasing the regioselectivity and decreasing the reactivity in these systems. In the cases of certain heteroaryl azides the formation of small but nontrivial amounts of the C-5 isomer shows the existence of a borderline between the two effects. 
Table 1. Relative yields $(\%)^{\mathrm{a}}$, m.p.s ${ }^{\mathrm{b}}$ and significant NMR spectroscopic datac for silylated triazoles 2a - i obtained by reaction of the corresponding azides $\mathbf{1 a}-\mathbf{i}$ in neat (trimethylsilyl)acetylene

\begin{tabular}{|c|c|c|c|c|c|c|c|}
\hline Entries & Azides & $t / d d$ & Triazoles & M.p.T/ ${ }^{\circ} \mathrm{C}$ & $\delta \mathrm{C}-4^{\prime}$ & $\begin{array}{c}\delta \mathrm{C}-5^{\prime}(J \mathrm{C}- \\
\mathrm{H})^{\mathrm{e}} \\
\end{array}$ & $\delta \mathrm{H}^{-5}$ \\
\hline 1 & $\begin{array}{l}\text { 2-(Trifluoromethyl)phenyl azide } \\
\text { 1a }\end{array}$ & 40 & $2 \mathrm{a}(96)$ & $28-30$ & 146.5 & $131.8(194)$ & 7.75 \\
\hline 2 & $\begin{array}{l}\text { 3-(Trifluoromethyl)phenyl azide } \\
\text { 1b }\end{array}$ & 34 & $2 b(96)$ & $74-75$ & 148.4 & $27.4(192)$ & 8.00 \\
\hline 3 & $\begin{array}{l}\text { 4-(Trifluoromethyl)phenyl azide } \\
\text { 1c }\end{array}$ & 12 & 2c (97) & $93-95$ & 148.5 & $127.3(191)$ & 8.02 \\
\hline 4 & 4 2-Fluorophenyl azide $\mathbf{1 d}$ & 20 & $2 \mathrm{~d}(97)$ & $34-35$ & 147.0 & $125.2(194)$ & 8.05 \\
\hline 5 & 5 3-Fluorophenyl azide 1e & 23 & 2e (96) & Oil & 148.1 & $127.4(192)$ & 7.95 \\
\hline 6 & 4-Fluorophenyl azide $\mathbf{1 f}$ & 18 & $2 f(97)$ & $98-99$ & 147.7 & $127.6(192)$ & 7.96 \\
\hline 7 & $\begin{array}{l}\text { 5-(Trimethylsilyl)-2- } \\
\text { selenophenyl azide } \mathbf{1 g}\end{array}$ & 10 & $2 \mathrm{~g}(96)$ & $69-71$ & 147.9 & $127.7(192)$ & 7.87 \\
\hline 8 & $\begin{array}{l}\text { 1-(5-Azido-2-thienyl)-1- } \\
\text { ethanone } \mathbf{1 h}\end{array}$ & 6 & $2 \mathrm{~h}(97)$ & $202-204$ & 148.6 & $128.0(194)$ & 7.90 \\
\hline 9 & $\begin{array}{l}\text { 1-(5-Azido-2-thienyl)-2,2,2- } \\
\text { trifluoro-1-ethanone } \mathbf{1 i}\end{array}$ & 5 & $2 \mathrm{i}(97)^{f}$ & $158-160$ & 148.9 & $127.8(194)$ & 7.98 \\
\hline
\end{tabular}

${ }^{a}$ Isolated yields based on starting azides after elimination of the excess solvent/reagent. ${ }^{b}$ All m.p.s are uncorrected. ${ }^{c}$ Measured in $\mathrm{CDCl}_{3}, \mathrm{CHCl}_{3}$ internal standard.

${ }^{d}$ Approximate reaction times (days) corresponding to complete consumption of starting azides. ${ }^{e}$ $J \pm 1 \mathrm{~Hz}$. $f$ Accompanied by small amounts of the C-5 silylated regioisomer, 2i' (1.5\%), m.p. 98$100^{\circ} \mathrm{C}, \mathrm{H}-4 ’ 7.81 \mathrm{ppm}$. 
Table 2. ${ }^{13} \mathrm{C}$ NMR spectroscopic data for triazoles $2 \mathbf{a}-\mathbf{i}^{\mathrm{a}}$

\begin{tabular}{|c|c|c|c|c|c|c|c|c|c|}
\hline Triazole & C-1 & $\mathrm{C}-2\left(J_{\mathrm{C}-\mathrm{H}}\right)$ & $\mathrm{C}-3$ & C-4 & C-5 & C-6 & $\mathrm{CF}_{3}$ & $\mathrm{SiMe}_{3}$ & Others \\
\hline $2 a$ & 135.2 & $126.3(32)^{b}$ & $\begin{array}{l}127.4 \\
(167)\end{array}$ & $\begin{array}{l}130.4 \\
(166)\end{array}$ & $\begin{array}{l}133.2 \\
(166)\end{array}$ & $\begin{array}{l}129.2 \\
(166)\end{array}$ & $\begin{array}{l}122.9 \\
(273)^{b}\end{array}$ & -0.9 & \\
\hline $2 \mathbf{b}$ & 137.8 & $118.0(165)$ & $\begin{array}{l}132.6 \\
(33)^{b}\end{array}$ & $\begin{array}{l}124.2 \\
(167)\end{array}$ & $\begin{array}{l}130.8 \\
(166)\end{array}$ & $\begin{array}{l}125.4 \\
(168)\end{array}$ & $\begin{array}{l}123.7 \\
(273)^{b}\end{array}$ & -0.8 & \\
\hline $2 c$ & 139.9 & $121.0(167)$ & $\begin{array}{l}127.4 \\
(162)\end{array}$ & $\begin{array}{l}130.8 \\
(33)^{b}\end{array}$ & $\begin{array}{l}127.4 \\
(162)\end{array}$ & $\begin{array}{l}121.0 \\
(167)\end{array}$ & $\begin{array}{l}124.0 \\
(272)^{b}\end{array}$ & -0.8 & \\
\hline 2d & 125.5 & $153.5(251)^{b}$ & $\begin{array}{l}117.0 \\
(165 ; 20 \\
\left.{ }^{b}\right)\end{array}$ & $\begin{array}{l}130.1 \\
(167)\end{array}$ & $\begin{array}{l}125.2 \\
(166)\end{array}$ & $\begin{array}{l}130.0 \\
(166)\end{array}$ & & -1.1 & \\
\hline $2 e$ & 138.6 & $\begin{array}{l}108.8 \\
\left.26^{b}\right)\end{array}$ & $\begin{array}{l}163.4 \\
(248)\end{array}$ & $\begin{array}{l}115.7 \\
(168 ; \\
\left.22^{b}\right)\end{array}$ & $\begin{array}{l}131.5 \\
(166 ; 8.5 \\
\left.{ }^{b}\right)\end{array}$ & $\begin{array}{l}116.4 \\
(166)\end{array}$ & -0.8 & & \\
\hline $2 f$ & 133.7 & $\begin{array}{l}122.9 \\
\left.9^{b}\right)\end{array}$ & $\begin{array}{l}117.4 \\
(165 ; \\
\left.23^{b}\right)\end{array}$ & $\begin{array}{l}162.5 \\
(249)^{b}\end{array}$ & $\begin{array}{l}117.4 \\
(165 ; \\
\left.23^{b}\right)\end{array}$ & $\begin{array}{l}122.9 \\
\left(165 ; 9^{b}\right)\end{array}$ & -0.9 & & \\
\hline $2 g$ & 146.8 & $120.7(165)$ & $\begin{array}{l}135.0 \\
(165)\end{array}$ & $146.8 ?$ & & & & -0.85 & 0.4 \\
\hline $2 h$ & 140.9 & $117.9(171)$ & $\begin{array}{l}132.1 \\
(170)\end{array}$ & 145.4 & & & & & -0.9 \\
\hline $2 \mathbf{i}$ & 132.4 & $117.8(168)$ & $\begin{array}{l}136.7 \\
(17 ; \quad 43 \\
\left.{ }^{b}\right)\end{array}$ & 149.3 & & & $\begin{array}{l}116.5 \\
(290)^{b}\end{array}$ & -1.0 & $\begin{array}{l}173.9 \\
(37)^{b}\end{array}$ \\
\hline
\end{tabular}

${ }^{a}$ In order to obtain as far as possible uniform and reliable chemical shifts $(\delta)$ and coupling constants $(J)$ the spectra are recorded for dilute solutions (ca. 10\%, wt) in $\mathrm{CDCl}_{3}$ and TMS as internal standard. Spectra of compounds 2a-f were recorded at $75.4 \mathrm{MHz}$ and 2 g-i at $50.3 \mathrm{MHz} .^{b}$ JC-F. 


\section{Experimental Section}

General Procedures. Instrumentation - Melting points were determined on a Kofler hotstage apparatus and are uncorrected. IR spectra were recorded on a Perkin-Elmer Model 257 instrument in film. ${ }^{1} \mathrm{H}$ NMR and ${ }^{13} \mathrm{C}$ NMR spectra were recorded on a Varian Gemini 200 (200 and $50 \mathrm{MHz}$, respectively) or 300 (300 and $75 \mathrm{MHz}$, respectively). J-values are given in $\mathrm{Hz}$. Mass spectra were recorded on VG7070E instruments using electron impact ionisation.

The 2-selenophenyl azide $1 \mathrm{~g}$ has been obtained following the procedure described for compound 1m 7a and was characterized by ${ }^{1} \mathrm{H}-,{ }^{13} \mathrm{C}-\mathrm{NMR}$, IR and exact mass spectra.

Materials - Aryl azides 1a - $\mathbf{f}$ were prepared from diazonium compounds and azide ion in alkaline medium according to the general procedure described by Smith and co-workers. ${ }^{11}$ Azidothiophenes $\mathbf{1 h}$ and $\mathbf{1 i}$, were prepared by selective bifunctionalization of the corresponding dibromothiophene. ${ }^{12}$ (Trimethylsilyl)acetylene was purchased from Aldrich Chimica Italiana and degassed with nitrogen before the use.

Caution - Hydrazoic acid and organic azides are potentially explosive! This category of compounds has recently been subjected to risk evaluation. ${ }^{13}$ Explosions may be expected by handling phenyl or tosyl azide, although we experienced no problems in handling basic solutions of sodium azide or solid organic azides, which may be stored at $-18{ }^{\circ} \mathrm{C}$ indefinitely.

5-(Trimethylsilyl)-2-selenophenyl azide (1g). (69\%); Colourless oil; $v_{\max } / \mathrm{cm}^{-1} 2980,2130$, 1465, 1260, 955 and 850; $\delta \mathrm{H}\left(200 \mathrm{MHz} ; \mathrm{CDCl}_{3}\right) 7.17(1 \mathrm{H}, \mathrm{d}, J 3.8 \mathrm{H}-4), 6.70(1 \mathrm{H}, \mathrm{d}, J 3.8 \mathrm{H}-3)$ and $0.30(9 \mathrm{H}, \mathrm{s}) ; \delta \mathrm{H}\left(50.3 \mathrm{MHz} ; \mathrm{CDCl}_{3}\right)$ 151.3, 143.6, $136.2(\mathrm{~J} 163.4), 119.6(\mathrm{~J} 164.6)$ and 0.48(q); m/z 245 (M+, 4.5\%), 230 (M-15, 0,9), 217 (M-28, 23.9), 189 (9.0), 163 (0.5), 138 (5.5), 73 (100) and 45 (35.8); Found: $\mathrm{M}+, 244.98870 \mathrm{C}_{7} \mathrm{H}_{11} \mathrm{~N}_{3} \mathrm{SeSi}$ requires: M, 244.98875.

\section{Reactions of aryl azides $1 \mathrm{a}-\mathrm{f}$ and heteroaryl azides $1 \mathrm{~g}-\mathrm{i}$ with (trimethylsilyl)acetylene at} $25^{\circ} \mathrm{C}$. General procedure

A solution of the azide in neat (trimethylsilyl)acetylene (1:10 molar ratio) was allowed to react in a screw cap tube for the appropriate time, until tlc showed the absence of the starting azide. The solvent-reagent was removed under vacuum and the residue washed with pentane and then characterized.

The following new triazoles were obtained: 1-[2-(trifluoromethyl)phenyl]-4-(tri-methylsilyl)1H-1,2,3-triazole (2a). (96\%), mp 28-30 ${ }^{\circ} \mathrm{C} ; v_{\max } / \mathrm{cm}^{-1} 3140,2980,1630,1325,1250,1180,1$ 140 and 845; $\delta \mathrm{H}\left(300 \mathrm{MHz} ; \mathrm{CDCl}_{3}\right) 7.94(1 \mathrm{H}, \mathrm{m}, \mathrm{H}-3), 7.75(1 \mathrm{H}, \mathrm{q}, J \mathrm{H}-\mathrm{F} 1.1, \mathrm{H}-5$ '), $7.66(1 \mathrm{H}$, 
m, H-5), $7.60(1 \mathrm{H}, \mathrm{m}, \mathrm{H}-4), 7.45(1 \mathrm{H}, \mathrm{m}, \mathrm{H}-6)$ and $0.39(9 \mathrm{H}, \mathrm{s}) ; \mathrm{m} / \mathrm{z} 285(0.6 \%, \mathrm{M}+), 257$ (19,3\%, M-N $), 242$ (14.1), 161 (17.9), 146 (53.0), 145 (33.8), 140 (14.1), 126 (28.0), 116 (29.2), 115 (86.4), 81 (43.0), 77 (100), 73 (23.7), 49 (14.7), 45 (15.2) and 43 (16.9) (Found: C, 50.50; H, 4.95; N, 14.75\%. $\mathrm{C}_{12} \mathrm{H}_{14} \mathrm{~F}_{3} \mathrm{~N}_{3}$ Si requires $\mathrm{C}, 50.51 ; \mathrm{H}, 4.95 ; \mathrm{N}, 14.73 \%$ ).

1-[3-(Trifluoromethyl)phenyl]-4-(trimethylsilyl)-1H-1,2,3-triazole (2b). (96\%), mp 74-75 ${ }^{\circ} \mathrm{C}$; $v_{\max } / \mathrm{cm}^{-1} 3140,2980,1500,1330,1210,1180,1140$ and 850; $\delta \mathrm{H}\left(300 \mathrm{MHz} ; \mathrm{C}_{6} \mathrm{H}_{6}\right) 7.40(1 \mathrm{H}$, bs, H-2), 7.25 (1H, bd, H-4), 7.00 (1H, s, H-5'), 6.95 (1H, bd, H-6), $6.60(1 \mathrm{H}, \mathrm{bt}, \mathrm{H}-5)$ and $0.20(9 \mathrm{H}$, s); m/z 285 (0.6\%, M+), 270 (3.0\%, M-CH $), 257$ (28.5\%, M-N $), 243$ (49.6), 242 (100), 227 (19.1), 145 (16.0), 126 (44.3), 77 (38.8), 73 (28.8), 49 (14.7), 45 (15.5) and 43 (20.6) (Found: C, $50.50 ; \mathrm{H}, 4.94 ; \mathrm{N}, 14.69 \%)$.

1-[4-(Trifluoromethyl)phenyl]-4-(trimethylsilyl)-1H-1,2,3-triazole (2c). (97\%), mp 93-95 ${ }^{\circ} \mathrm{C}$; $v_{\max } / \mathrm{cm}^{-1} 3130,2980,1630,1330,1210,1180,1160$ and $835 ; \delta \mathrm{H}\left(300 \mathrm{MHz} ; \mathrm{CDCl}_{3}\right) 8.03(1 \mathrm{H}, \mathrm{s}$, H-5'), 7.91 (2H, bd, J 8.7, H-2 and H-6), 7.78 (2H, bd, $J$ 8.7, H-3 and H-5) and 0.39 (9H, s); m/z 285 (0.6\%, M+), 270 (3.0\%, M-CH $), 257$ (29.9\%, M-N 2 ), 244 (17.1), 243 (66.0), 242 (100), 145 (18.9), 77 (22.9), 73 (34.3), 45 (18.6) and 43 (23.4) (Found: C, 50.50; H, 4.90; N, 14.75\%). 1-(2-Fluorophenyl)-4-(trimethylsilyl)-1H-1,2,3-triazole (2d). (97\%), mp 34-35 ${ }^{\circ} \mathrm{C} ; v_{\max } / \mathrm{cm}^{-1}$ 3125, 2990, 2920, 1540, 845, 765 and 680; $\delta \mathrm{H}\left(300 \mathrm{MHz} ; \mathrm{CDCl}_{3}\right) 8.05$ (1H, s, JH-F2.9, H-5'), 7.94 (1H, m, H-6), 7.41 (1H, m, H-4), 7.32 (1H, m, H-5), 7.32 (1H, m, H-6), 7.27 (1H, m, H-2) and $0.34(9 \mathrm{H}), \mathrm{s}) ; \mathrm{m} / \mathrm{z} 235$ (1.0\%, M+), 220 (1.7\%, M-Me), 207 (18.6, M-N $), 192$ (14.1), 161 (100), 139 (17.0), 130 (65.7), 103 (18.4), 91 (14.9), 89 (18.7), 77 (65.6), 73 (16.9), 47 (21.2) and 43 (14.6) (Found: C, 56.13; H, 5.98; N, 17.86\%. $\mathrm{C}_{11} \mathrm{H}_{14} \mathrm{FN}_{3} \mathrm{Si}$ requires $\mathrm{C}, 56.14 ; \mathrm{H}, 6.00 ; \mathrm{N}$, $17.86 \%)$.

1-(3-Fluorophenyl)-4-(trimethylsilyl)-1H-1,2,3-triazole (2e). (96\%), Colourless oil; $v_{\max } / \mathrm{cm}^{-1}$ 3190, 2990, 1620, 1260, 850, 765 and 685; $\delta \mathrm{H}\left(300 \mathrm{MHz} ; \mathrm{CDCl}_{3}\right) 7.95(1 \mathrm{H}, \mathrm{s}, \mathrm{H}-5$ ') $7.55(1 \mathrm{H}$, m, H-6), $7.52(1 \mathrm{H}, \mathrm{m}, \mathrm{H}-5), 7.47(1 \mathrm{H}, \mathrm{m}, \mathrm{H}-2), 7.13(1 \mathrm{H}, \mathrm{m}, \mathrm{H}-4)$ and $0.38\left(9 \mathrm{H}, \mathrm{s}, \mathrm{SiMe}_{3}\right) ; \mathrm{m} / \mathrm{z}$ 235 (1.0\%, M+), 220 (1.7\%, M-Me), 207 (7.6, M-N $), 192$ (100), 161 (100), 95 (5.5), 77 (6.4), 73 (8.5), 45 (5.6) and 43 (7.5) (Found: C, 56.14; H, 5.96; N, 17.83\%).

1-(4-Fluorophenyl)-4-(trimethylsilyl)-1H-1,2,3-triazole (2f). (97\%), mp 98-99 ${ }^{\circ} \mathrm{C} ; v_{\max } / \mathrm{cm}^{-1}$ 3060, 2970, 2920, 1520, 1250, 840 and 740; $\delta \mathrm{H}\left(300 \mathrm{MHz} ; \mathrm{CDCl}_{3}\right) 7.96\left(1 \mathrm{H}, \mathrm{s}, \mathrm{H}-5^{\prime}\right) 7.71(2 \mathrm{H}$, m, $J$ 9.1 and JH-F 4.6, H-2 and H-6), 7.18 (2H, m, J 9.1 and JH-F 8.1, H-3 and H-5) and 0.37 (9H, s, $\left.\mathrm{SiMe}_{3}\right) ; \mathrm{m} / \mathrm{z} 235$ (1.0\%, M+), 220 (2\%, M-Me), 207 (18.1, M-N $), 193$ (34.1), 192 (100), 95 (14.0), 73 (20.9), 45 (15.2) and 43 (19.2) (Found: C, 56.14; H, 5.99; N, 17.85\%). 
4-(Trimethylsilyl)-1-[5-(trimethylsilyl)-2-selenophenyl]-1H-1,2,3-triazole (2g). (96\%), mp $69-71{ }^{\circ} \mathrm{C} ; v_{\max } / \mathrm{cm}^{-1} 2990,1630,1325,1140,845,770$ and $700 ; \delta \mathrm{H}\left(200 \mathrm{MHz} ; \mathrm{CDCl}_{3}\right) 7.87(1 \mathrm{H}$, s, H-5'), 7.38 (1H, d, J 3.8, H-4), $7.36(1 \mathrm{H}, \mathrm{d}, J 3.8, \mathrm{H}-3), 0.37(9 \mathrm{H}, \mathrm{s})$ and $0.34(9 \mathrm{H}, \mathrm{s}) ; \mathrm{m} / \mathrm{z} 343$ (0.4\%, M+), 315 (21.9\%, M-N 2$), 300$ (57.9), 298 (19.8), 73 (100), 45 (18.2) and 43 (12.6) (Found: C, 42.09; H, 6.15; N, 12.25\%. $\mathrm{C}_{12} \mathrm{H}_{21} \mathrm{~N}_{3} \mathrm{SeSi}_{2}$ requires $\mathrm{C}, 42.10 ; \mathrm{H}, 6.18 ; \mathrm{N}, 12.27 \%$ ). 1-\{5-[4-(Trimethylsilyl)-1H-1,2,3-triazol-1-yl]-2-thienyl\}-1-ethanone (2h). (97\%), mp 202$04{ }^{\circ} \mathrm{C} ; v_{\max } / \mathrm{cm}^{-1}$ 3140, 2980, 1650, 1250 and 845; $\delta \mathrm{H}\left(200 \mathrm{MHz} ; \mathrm{CDCl}_{3}\right) 7.90$ (1 H, s, H-5'), $7.62(1 \mathrm{H}, \mathrm{d}, J$ 4.1, H-4), 7.30 (1 H, d, J 4.1, H-3), $2.58(3 \mathrm{H}, \mathrm{s})$ and $0.37(9 \mathrm{H}, \mathrm{s}) ; \mathrm{m} / \mathrm{z} 237$ (35.4\%, M-N $\mathrm{N}_{2}$ ), 222 (100), 182 (5.5), 83 (22.8), 73 (52.1) and 55 (5.0) (Found: C, 49.75; H, 5.70; N, $15.80 \% . \mathrm{C}_{11} \mathrm{H}_{15} \mathrm{~N}_{3} \mathrm{OSSi}$ requires $\left.\mathrm{C}, 49.78 ; \mathrm{H}, 5.70 ; \mathrm{N}, 15.83 \%\right)$.

2,2,2-Trifluoro-1-\{5-[4-(trimethylsilyl)-1H-1,2,3-triazol-1-yl]-2-thienyl\}-1-ethanone

(2i). (97\%), mp 158-60 ${ }^{\circ} \mathrm{C} ; v_{\max } / \mathrm{cm}^{-1} 3170,3130,3120,3100,1705,1185,1150$ and 850(SiMe $) ; \delta \mathrm{H}$ $\left(200 \mathrm{MHz} ; \mathrm{CDCl}_{3}\right) 7.98$ (1 H, s, H-5'), 7.91 (1H, dq, J 4.4 and JHF1.47, H-4), 7.38 (1 H, d, J 4.4, H-3) and 0.39 (9H,s, $\left.\mathrm{SiMe}_{3}\right) ; \mathrm{m} / \mathrm{z} 304$ (1.3\%, M-Me), 291 (19.5\%, M-N $), 276$ (82.4), 149 (10.6), 83 (37.8), 77 (15.1), 73 (100), 45 (16.4) and 43 (20.4) (Found: C, 41.35; H, 3.80; N, $13.10 \% . \mathrm{C}_{11} \mathrm{H}_{12} \mathrm{~F}_{3} \mathrm{~N}_{3} \mathrm{OSSi}$ requires $\left.\mathrm{C}, 41.37 ; \mathrm{H}, 3.79 ; \mathrm{N}, 13.16 \%\right)$.

2,2,2-Trifluoro-1-\{5-[5-(trimethylsilyl)-1H-1,2,3-triazol-1-yl]-2-thienyl\}-1-ethanone (2i'). (1.5\%), mp 98-100 ${ }^{\circ} \mathrm{C} ; v_{\max } / \mathrm{cm}^{-1} 1700,1180,1150$ and 850; $\delta \mathrm{H}\left(200 \mathrm{MHz} ; \mathrm{CDCl}_{3}\right) 7.95(1 \mathrm{H}$, dq, J 4.15 and JHF 1.5, H-4), 7.81 (1 H, s, H-4'), 7.32 (1 H, d, J 4.15, H-3) and 0.34 (9H, s, $\left.\mathrm{SiMe}_{3}\right) ; \mathrm{m} / \mathrm{z} 291$ (32.1\%, M-N $), 277$ (15.9), 276 (97.8), 149 (11.1), 86 (13.9), 84 (21.2), 83 (23.8), 77 (11.0), 73 (100), 45 (20.8) and 43 (14.0) (Found: C, 41.35; H, 3.75; N, 13.15\%).

Crystallography of (2i) and (2l). 2,2,2-Trifluoro-1-\{5-[4-(trimethylsilyl)-1H-1,2,3-triazol-1yl]-2-thienyl\}-1-ethanone 2i and 1-phenyl-4-(trimethylsilyl)-1H-1,2,3-triazole 2l single crystals were investigated.

Monoclinic, space group $\mathrm{P} 2(1) / \mathrm{n} ; \mathrm{a}=6.6365(15), \quad \mathrm{b}=31.662(8), \quad \mathrm{c}=7.1733(17) \quad \AA$, $\beta=90.068(6)^{\circ}, Z=4, V=1507.3(6) \AA 3$ was $2 \mathbf{i}$.

Monoclinic, space group P2(1)/c; $\quad \mathrm{a}=15.674(3), \quad \mathrm{b}=7.3358(14), \quad \mathrm{c}=11.033(2) \quad \AA$, $\beta=101.508(4)^{\circ}, Z=4, V=1243.2(4) \AA 3$ was $2 \mathbf{l}$.

The diffraction experiments were carried out at $293 \mathrm{~K}$ on a Bruker AXS SMART 2000 CCD area detector diffractometer. Graphite-monochromatyzed Mo-Ka $(\lambda=0.71073 \AA)$ radiation was used with generator working at $50 \mathrm{KV}$ and $40 \mathrm{~mA}$. 
The intensity data were collected using the $\omega$-scan technique within the limits $2.91<2 \theta<50.04^{\circ}$.

9426 reflections were read, of which 2641 independent reflections, 830 observed for $\mathrm{I}>2 \sigma$ (I).

Final R factors: $\mathrm{R} 1=0.0775, \mathrm{wR} 2=0.1689$ for $2 \mathbf{i}$.

22411 reflections were read, of which 2187 independent reflections, 762 observed for I $>2 \sigma$ (I).

Final R factors: R1 $=0.0529$, wR2 $=0.1023$ for $2 \mathbf{l}$.

The collected frames were processed by SAINT software for integration; an absorption correction was applied together with merging (SADABS $)^{14}$.

The structures were solved in the space group P2(1)/n for $2 \mathbf{i}$ and P2(1)/c for $2 \mathbf{l}$ by direct methods and difference Fourier maps and subsequently refined by full-matrix least-squares against Fo using the SHELXTL V.5.1 suite of programs. ${ }^{15}$ All non-hydrogen atoms were refined anisotropically. Hydrogen atoms were first identified in difference Fourier maps, then included in idealized positions and refined riding on their carrier atoms with isotropic thermal parameters 1.2 times those of the pertinent atoms. The final difference Fourier maps were featureless.

\section{Acknowledgements}

We gratefully acknowledge financial support by Ministero dell'Università e della Ricerca Scientifica e Tecnologica (MURST) and partial support from the "Progetto di Finanziamento Triennale, Ateneo di Bologna".

\section{References and Notes}

1. Zanirato, P. J. Chem. Soc., Perkin Trans. 1 1991, 2789.

2. Fan, W-Q.; Katritzky, A. R. Rees, C. W.; Scriven, E. F. V. Comprehensive Heterocycl. Chem. II, Storr R. C., Ed., Pergamon Press: Oxford, 1996; Vol. 4, p 1.

3. Wamhoff H., In Comprehensive Heterocycl. Chem., Katritzky A. R., Rees C. W.; Post K. T., Eds., Pergamon Press: Oxford, 1984; Vol. 5, Part 4.A, p 669.

4. (a) March J. Advanced Organic Chemistry, $4^{\text {th }}$ Edn; Wiley: N.Y., 1992; p 836. (b) Gilchrist, T. L.; Gymer G. E. Adv. Heterocycl. Chem. 1974, 16, 33. (c) L’abbè, G. Chem. Rev. 1969, $69,345$.

5. (a) Gothelf, K.V.; Jorgensen, K.A. Chem. Rev. 1998, 98, 863. (b) 1,3-Dipolar Cycloaddition 
Chemistry, Padwa A., Ed.; Wiley-Interscience: New York, 1984; Vols. 1-2. (c) Bastide, J.; Henri-Rousseau O. The Chemistry of the C-C Triple Bond, Patai S.; Wiley, 1978, Cap. 11, 447. (d) Huisgen, R.; Szeimies, G.; Mobius, L. Chem. Ber. 1967, 100, 2494. (e) Huisgen, R.; Knorr, R.; Mobius, L.; Szeimies, G. Chem. Ber. 1965, 98, 4014.

6. (a) Halevi, E. A. Ed., Orbital Symmetry and Reaction Mechanism, Springer-Verlag: Berlin, 1992. (b) Fleming I. Frontier Orbitals and Organic Chemical Reactions, WileyInterscience: London, 1976. (c) Huisgen, R. Angew. Chem., Int. Ed. 1963, 2, 565 . (d) idem ibid. 633. (e) J. Org. Chem. 1976, 41, 403.

7. (a) Domingo, L. R. Eur. J. Org. Chem. 2000, 2265. (b) Chandra, A. K.; Uchimaru, T.; Nguyen, M. T. J. Chem. Soc. Perkin Trans. 2 1999, 2117.

8. Davies, D.; Spagnolo, P.; Zanirato, P. J. Chem. Soc., Perkin Trans.1 1995, 613.

9. Gronowitz, S.; Zanirato, P. J. Chem. Soc., Perkin Trans.1 1994, 1815.

10. Spinelli, D.; Zanirato, P. J. Chem. Soc., Perkin Trans. 1 1993, 1129.

11. Preliminary in vitro test of some 1-heteroaryl C-4 silylated 1,2,3-triazoles have been recently evaluated for confirmatory and advanced screening by TAACF (Tubercolosis Antimicrobial Acquisition \& Coordinating Facility) laboratory (Birmingam, Alabama).

12. Fifolt, M. J.; Sojka, S. A.; Wolfe, R. A.; Hojnicki, D. S.; Bieron, J. F.; Dinan, F. J. J. Org. Chem. 1989, 54, 3019.

13. Charton, M. Progres. Phys. Org. Chem. 1981, 13, 119.

14. Adcock, W. ; Khor, T-C. J. Am. Chem. Soc. 1978, 100, 7799.

15. Coulson, D. R. J. Am. Chem. Soc. 1976, 98, 3111.

16. Ernst, L.; Wray, V.; Chertkov, V. A.; Sergeyev, M. J. Mag. Res. 1977, 25, 123.

17. Gronowitz, S.; Johson, I.; Hörnfeldt, A.-B. Chemica Scripta 1975, 7, 76 and 111.

18. Chachaty, C.; Forchioni, A.; Virlet J. Can. J. Chem. 1975, 53, 648.

19. (a) Weigert, F. J.; Roberts, J. D. J. Am. Chem. Soc. 1971, 93, 2361. (b) Page T. F. Mol. Phys. 1967, 13, 523.

20. For recent studies on this topics: (a) Funicello, M.; Spagnolo, P.; Zanirato, P. Acta Chem. Scand., Ser. B 1993, 47, 231. (b) Becher, J.; Dehaen, W. Acta Chem. Scand., Ser. B 1993, 47, 277. (c) Zanirato, P. Gazz. Chim. Ital. 1997, 127, 339. (d) Valenti, F.; Zanirato, P. J. Chem. Soc. Perkin Trans. 1 1999, 623.

21. Smith, P. A.; Brown, B. B. J. Am. Chem. Soc. 1951, 73, 2438.

22. Spagnolo, P. ; Zanirato, P. J. Chem. Soc. Perkin Trans.1 1996, 963.

23. (a) Bollinger, F. W.; Tuma, L. D. Synlett 1996, 407. (b) TSCA New chemicals program (NCP) chemical categories: http://www.epa.gov/oppt/newchems/chemcat.htm.

24. Sheldrick, G. M., SADABS: Program for Absorption Correction, 1996, University of Göttingen. 
25. Sheldrick, G. M., SHELXTL 5.1/NT. An integrated system for solving, refining and displaying crystal structures from diffraction data, Bruker AXS Inc., Madison, WI, 1997. 